
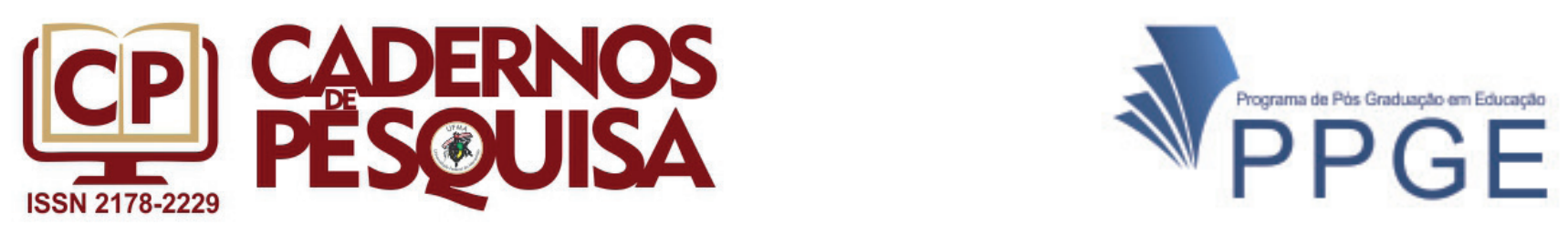

\title{
Fatores determinantes no uso de tecnologias digitais de informação e comunicação no ensino superior
}

\author{
Determining factors for the use of digital information and \\ communication technologies in higher education
}

\section{Factores determinantes para el uso de las tecnologías digitales de información y comunicación en la enseñanza superior}

\author{
Pollyanna Roberta Campelo Görgens \\ (iD) http://orcid.org/0000-0001-6478-9889 \\ Paulo César de Resende Andrade \\ (iD) $h t t p: / / o r c i d . o r g / 0000-0002-7865-8174$
}

\begin{abstract}
Resumo: As Tecnologias Digitais de Informação e Comunicação (TDICs) são parte dos métodos inovadores previstos em projetos pedagógicos de cursos de graduação mais recentes. Porém, diante de um quadro de professores procedentes de escolas tradicionais, conhecer as variáveis que trazem impacto no uso destas tecnologias para o ensino e aprendizagem podem contribuir no direcionamento de futuras medidas de meIhorias e políticas de incentivos. Este estudo tem como objetivo investigar os fatores que determinam uso das TDICs por professores da Faculdade de Medicina de Diamantina (FAMED) da Universidade Federal dos Vales do Jequitinhonha e Mucuri (UFVJM). Foi realizado um estudo de campo observacional transversal a partir de questionários aplicados a população total composta por 48 docentes efetivos em exercício. Destes, 42 participaram do estudo. Foi verificada a existência de associação entre as variáveis estudadas para determinar quais delas teriam impacto na quantidade de ferramentas de TDICs usadas pelos docentes. Os testes estatísticos foram realizados considerando um nível de significância de $5 \%$ para verificar associações entre as variáveis. Concluiu-se que a existência de uma aptidão prévia dos futuros professores com as tecnologias, antes do ingresso na carreira de magistério, é um fator decisivo para uma maior adequação das atividades desenvolvidas ás necessidades do curso. A motivação pessoal, a impressão de sentir apoio institucional e as características pessoais do professor são os principais fatores determinantes para o maior uso de TDICs na docência.
\end{abstract}

Palavras-chave: Educação Médica. Tecnologia Educacional. Tecnologia da Informação. Faculdades de Medicina

Abstract: The Digital Information and Communication Technologies (DICTs) are part of the innovative methods described on pedagogical project of new graduation courses. However, facing with a group of teachers coming from traditional schools, knowing the variables that have impact on the use of these education technologies can contribute to guide next improvement measures and incentive policies. This study aims to inves- 
tigate the factors that determine the use of DICTs by teachers of Diamantina Faculty of Medicine (FAMED) from Vale do Jequitinhonha e Mucuri Federal University (UFVJM). A cross-sectional observational study was carried out based on questionnaires applied to the total population composed of 48 effective faculty members. Of those, 42 participated in the study. It was verified the existence of an association between the variables studied to determine which of them would have an impact on the amount of DICTs tools used. Statistical tests were perform considering $5 \%$ significance level to verify associations between the variables. It was concluded that the existence of previous aptitude of the future teachers with technologies, before starting the teaching career, is a decisive factor for a better adaptation to the course needs. The personal motivation, the institutional support feeling and the personal characteristics of the teacher are the main determining factors for the greater use of DICTs in teaching.

Keywords: Medical Education. Educational Technology. Information Technology. Medical Schools.

Resumen: Las Tecnologías Digitales de Información y Comunicación (TDICs) son parte de los métodos innovadores previstos en los proyectos pedagógicos de cursos de graduación más recientes. Sin embargo, ante un cuadro de profesores procedentes de escuelas tradicionales, conocer las variables que traen impacto en el uso de estas tecnologías para la enseñanza y el aprendizaje pueden contribuir en la dirección de futuras medidas de mejoras y políticas de incentivos. Este estudio tiene como objetivo investigar los factores que determinan el uso de las TDIC por profesores de la Facultad de Medicina de Diamantina (FAMED) de la Universidad Federal dos Vales do Jequitinhonha e Mucurí (UFVJM). Se realizó un estudio de campo observacional transversal a partir de cuestionarios aplicados a la población total compuesta por 48 docentes efectivos en ejercicio. De ellos, 42 participaron del estudio. Se verificó la existencia de asociación entre las variables estudiadas para determinar cuáles de ellas tendrían impacto en la cantidad de herramientas de TDIC utilizadas por los docentes. El software R se utilizó para el análisis gráfico y estadístico. Las pruebas estadísticas se realizaron considerando un nivel de significancia $5 \%$ para verificar las asociaciones entre las variables. Se concluyó que la existencia de una aptitud previa de los futuros profesores con las tecnologías, antes del ingreso en la carrera de maestría, es un factor decisivo para una mayor adecuación de las actividades desarrolladas a las necesidades del curso. La motivación personal, la impresión de sentir apoyo institucional y las características personales del profesor son los principales factores determinantes para el mayor el uso de TDIC en la docencia.

Palabras clave: Educación Médica. Tecnología Educativa. Tecnología de la información. Facultades de Medicina

\section{Introdução}

As Tecnologias Digitais de Informação e Comunicação (TDICs) são parte dos métodos de ensino previstos em projetos pedagógicos inovadores. Elas possuem o objetivo de auxiliar na construção de um cenário de prática virtual e compatível com a vivência do aluno estimulando o resgate de conceitos e associações prévias, facilitando o aprendizado. Ao incorporar as TDICs na atuação docente, é possível ministrar aulas mais motivadoras e estimular a autonomia do aluno em favor de uma educação reflexiva e problematizadora da realidade (PEREIRA et al., 2016).

Inicialmente, o termo utilizado para designar tecnologias vinculadas à área da informática era Tecnologias da Informação (TI) (DOWBOR, 2013). A expressão Tecnologias de Informação e Comunicação (TICs) surgiu a partir do final da década de 70, com 
o desenvolvimento da informática e da comunicação entre computadores. As TICs podem então ser definidas como um conjunto de tecnologias associadas com o processamento de informação, envio e recebimento de mensagens. Elas permitem uma nova forma de pensar e conviver (UNITED NATIONAL EDUCATIONAL, 2015). As formas que sugiram a partir da década de 90 passaram a se chamar Tecnologias Digitais de Informação e Comunicação (TDICs), pois o funcionamento se baseia na lógica binária, que consiste no fato de que toda informação deve ser expressa na forma de dois dígitos, zero ou um, também denominadas NTICs (Novas Tecnologias da Informação e da Comunicação) (ALONSO, 2002).

Atualmente, dentro do contexto da educação, as Tecnologias de Informação e Comunicação (TICs) fazem referência a qualquer tecnologia utilizada para apoiar e melhorar a aprendizagem dos alunos e desenvolver ambientes de aprendizagem e pode-se considerar, por exemplo, livros e filmes (MIRANDA, 2007). Já o termo TDICs envolve as tecnologias em que o computador é o instrumento de uso principal (LOPES, 2010).

Jean Piaget descreve a teoria de que o homem nasce com uma fascinante bagagem hereditária, porém não consegue emitir uma simples operação de pensamento. Assim, o conhecimento não nasce com o indivíduo, nem é dado pelo meio social. O sujeito constrói seu conhecimento na interação com o meio tanto físico como social. O construtivismo traz a ideia de que nada está pronto e que, da mesma forma, o conhecimento não é dado, em nenhuma instância, como algo terminado (BECKER, 1992).

De acordo com as Diretrizes Curriculares Nacionais (DCN) dos Cursos de Graduação em Medicina (BRASIL, 2014), uma das habilidades esperadas dos egressos envolve aprender a aprender, identificando conhecimentos prévios e formulando questões para a busca de respostas cientificamente consolidadas. Esta habilidade requer o manuseio dos recursos tecnológicos existentes em favor de sua formação e atualização.

Para atender a estas diretrizes são necessários docentes melhor preparados, remunerados e valorizados. Infelizmente não é o que acontece na maioria das instituições educacionais. As instituições terão relevância quando apostarem na educação com projetos pedagógicos atualizados, com metodologias atraentes, com materiais interessantes e com profissionais capacitados para gerenciar atividades de aprendizagem mais complexas e desafiadoras (MORAN, 2015).

Neste aspecto, as atividades de ensino à distância (EaD) têm ganhado espaço progressivo na educação superior, buscando facilitar a comunicação do estudante com o docente. $\mathrm{O}$ ambiente virtual de aprendizagem pode ser empregado como espaço de convergência, voltado para compartilhar as diversas vivências experimentadas pelos estudantes nos diferentes cenários (LAU et al., 2017).

Por outro lado, existe uma tradição oral e presencial de transmissão de conhecimento, com o ensino centrado no professor, o aluno como receptor passivo e a avaliação como reprodução do conteúdo em sala. Assim, o professor precisa reconhecer a tecnologia como recurso, atuando como mediador na construção do conhecimento para enfrentar determinada situação (PEREIRA et al., 2016). 
Entre os diversos aspectos relativos à inadequação da formação médica, o fator mais agravante diz respeito ao perfil do médico formado, centrado no modelo biologicista e na medicalização. O hospital é o centro de toda a formação médica sendo que hoje se sabe que a maior parte dos atendimentos médicos se resolvem sem necessidade de hospitalização. Assim, a partir de 2002, o Ministério da Saúde passou a fornecer patrocínio para as escolas de graduação em medicina que se dispuseram a aderir a projetos de mudança nos currículos de seus cursos, com enfoque nas necessidades de saúde da população e do SUS e na adoção de metodologias ativas de ensino-aprendizagem (OLIVEIRA et al., 2008).

A mudança de paradigma sobre a forma de educar é necessária, porém, diante de um quadro de professores procedentes de escolas tradicionais, pode haver resistência do uso de recursos didáticos modernos. É necessário incentivar a relação aluno-professor, fazendo com que a aprendizagem seja percebida como uma descoberta, evidenciando a contribuição da tecnologia neste processo.

Segundo Debald (2007), o professor deve buscar atualização contínua, como forma de possibilitar isto também aos seus alunos, por meio de aulas mais motivadoras e inovadoras. Porém, o uso das tecnologias em sala de aula é um processo que depende não só da vontade do docente. O gestor também deve incentivar oferecendo cursos de capacitação e assessoria pedagógica e tecnológica para potencialização do ensino.

Nos últimos anos, houve investimentos significativos em tecnologias para conectar alunos e professores no ensino presencial e a distância. Isto permitiu ampliar o conceito de aula, de espaço e de tempo, estabelecendo uma nova forma de estar junto, fisicamente e virtualmente. Fóruns, pesquisas em base de dados e jogos de simulação, são exemplos de estratégias que podem ser utilizadas dentro da proposta de aprendizado, construtivista.

A Stanford University (2016), nos Estados Unidos, oferece todo o suporte necessário para o uso das TDICs. A utilização pedagógica das TDICs é feita em espaço restrito a comunidade acadêmica local, porém alguns espaços em experimentação são abertos ao público, como é o caso do jogo Septris (2018), desenvolvido por médicos, para o ensino e tratamento da Sepse.

Neste jogo o participante pode examinar o paciente, solicitar exames, propor tratamentos e consultas com especialistas. Se bem-sucedido o paciente irá receber alta e um novo caso será proposto. Existe um tempo para realizar as ações e para que os exames fiquem prontos. Enquanto isso, condutas iniciais devem ser tomadas para que o paciente pare de descer na tela principal. Se estas ações são incorretas ocorre uma rápida piora e quando o paciente chega à base da tela ocorre o óbito. Este é um exemplo de adequada utilização das TDICs como ferramenta de ensino-aprendizagem na área médica.

As escolas conectadas podem fazer uma integração maior entre a sala de aula, as atividades práticas e os espaços virtuais de aprendizagem. Podem disponibilizar as informações básicas de cada assunto, atividade ou projeto num ambiente virtual e fazer atividades que se conectam com a vida no entorno a partir de tablets, celulares ou notebooks, dentro e fora da sala de aula. Apesar disso, mesmo em escolas com menos recursos tam- 
bém é possível desenvolver projetos significativos e relevantes para os alunos, ligados à comunidade, utilizando tecnologias simples como o celular (MORAN, 2015).

O WhatsApp (WHATSAPP, 2018) permite a criação de grupos de discussão entre professores e alunos. Porém assim como os recursos de videoconferência, depende de acesso do aluno a internet, que nem sempre está disponível em locais mais afastados.

Cada escola precisa definir um plano estratégico apoiando e capacitando coordenadores, professores e alunos para trabalhar mais com metodologias ativas e com currículos mais flexíveis. Muitas instituições contratam profissionais pouco atualizados, mal remunerados, sobrecarregados de atividades e de alunos e sem a disciplina necessária para gerenciar fóruns, prazos e atividades. O mesmo acontece com parte dos alunos, pouco autônomos, com deficiências na formação básica. $O$ ambiente digital para quem não está acostumado é confuso, distante, pouco intuitivo e desagradável. Ainda estamos avançando muito pouco em relação ao que é preciso (MORAN, 2015).

Historicamente os professores construíam sua própria maneira de ensinar e avaliar conforme suas vivências. A atenção dos alunos convergia para a experiência do profissional em casos bem-sucedidos que eram narrados nas aulas. Porém a docência no ensino superior é uma tarefa complexa, para a qual é necessário preparo. Apesar do conceito de professor como mediador da aprendizagem e de um espaço cada vez mais aberto para novas tecnologias e metodologias, o professor continua a "dar aula", no sentido de ser responsável por organizar conteúdos e mediar os processos de aprendizagem. As tecnologias interativas, podem proporcionar enriquecidas possibilidades, como receber e responder mensagens dos alunos, criar fóruns para discussão e favorecer os debates e pesquisas com textos, vídeos e outros documentos disponíveis na internet (CARLINI, 2008).

Felizmente, os ambientes de aprendizado estruturados e com muitas funcionalidades tem se tornado mais simples. Sistemas como o Eliademy (ELIADEMY, 2018) e KhanAcademy (KHANACADEMY, 2018) foram desenvolvidos para que o coordenador do curso, com mínima habilidade em TDICs, possa criar todo um módulo, sem treinamento prévio.

O projeto pedagógico da Faculdade de Medicina (FAMED), da Universidade Federal dos Vales do Jequitinhonha e Mucuri (UFVJM), pretende favorecer o uso destas tecnologias durante o desenvolvimento das unidades curriculares, cursos, seminários e treinamentos (UFVJM, 2017). Diante de um curso recente, ainda em fase estruturação, de um projeto pedagógico baseado em propostas inovadoras e do fato de que o uso de TDICs no ensino é opcional, conhecer as variáveis que trazem impacto no uso de tecnologias para o ensino e aprendizagem pode contribuir no direcionamento de futuras medidas de melhorias e políticas de incentivos.

Além das TDICs, as metodologias ativas de ensino e aprendizagem também são consideradas estratégias inovadoras utilizados pelo curso. Elas procuram favorecer a autonomia do discente para se auto gerenciar no processo de formação. $\mathrm{O}$ aluno precisa ter consciência de que sua participação é fundamental para que o aprendizado ocorra. Nessa nova postura, torna-se essencial ao docente assumir o papel de facilitador (UFVJM, 2017). 
Espera-se que os resultados deste estudo possam incentivar intervenções direcionadas para o que se mostra eficaz na população estudada. O referencial teórico estudado sobre o tema TDICs no ensino aborda a necessidade de intervenções envolvendo a revisão de políticas de incentivo, currículos, metodologias educativas e qualificação técnica e pedagógica dos docentes.

O objetivo desta pesquisa é investigar os fatores determinantes para o uso das Tecnologias Digitais de Informação e Comunicação (TDICs) no curso de Medicina da Universidade Federal dos Vales do Jequitinhonha e Mucuri (UFVJM) - Campus JK. São objetivos específicos: determinar o perfil demográfico e profissional dos docentes, analisar como os mesmos inserem as tecnologias em suas práticas pedagógicas, verificar como o projeto pedagógico do curso favorece o uso das tecnologias na docência e analisar as variáveis que determinam a utilização ou não desses instrumentos.

\section{Metodologia}

Foi realizado um estudo de campo observacional transversal sobre a utilização das TDICs pelos docentes da FAMED. Segundo Medronho et al. (2009), em um estudo observacional o pesquisador simplesmente observa a população e suas características, sem controlar a exposição ou alocação dos indivíduos em grupos, lançando mão de uma situação dada e observando os resultados. Nos estudos transversais a exposição ao fator é analisada no mesmo momento que o desfecho.

Foram aplicados questionários aos participantes. O critério de inclusão foi ser um docente efetivo da FAMED. Os critérios de exclusão foram estar em licença temporária ou em período férias. Os docentes que devolveram o questionário incompleto também foram excluídos do estudo. Todos os questionários devolvidos com as 15 perguntas respondidas, foram considerados na abordagem dos dados.

As perguntas do questionário foram elaboradas a partir dos objetivos propostos, das informações encontradas durante o estudo do referencial teórico e do contexto vivido na FAMED. Foi optado por propor respostas fechadas padrão, para maior uniformidade das respostas, favorecendo a análise estatística.

As perguntas de 1 a 7 e a pergunta 15 do questionário possuem o objetivo de determinar o perfil demográfico e profissional dos docentes. São perguntas sobre sexo, idade, formação, titulação, regime de trabalho, tempo de docência na FAMED. A pergunta 15 solicita aos participantes que assinalem TDICs usados no ambiente pessoal.

A pergunta 13 possui o objetivo de analisar como as tecnologias são inseridas nas práticas pedagógicas. Nela os participantes assinalaram quais TDICs eram utilizadas nas atividades de docência. As TDICs sugeridas no questionário envolvem níveis de complexidade mais simples como programas de apresentação e mais complexas como a criação de jogos de simulação de situações médicas.

As perguntas 7 a 12 e a pergunta 14 possuem o objetivo de verificar como o projeto pedagógico do curso favorece o uso das tecnologias na docência. Foram realizadas per- 
guntas como: "Você já realizou algum curso de capacitação em informática?" Caso positivo havia a opção de assinalar se o curso foi ou não realizado com incentivo da instituição. $A$ pergunta 8 questionava se as estratégias adotadas pelo curso de medicina favoreciam o uso de TDICs nas aulas e, em caso afirmativo, havia a opção adicional de descrever como favorece. Esta foi a única pergunta que permitia uma resposta livre. Foi questionado ainda se há a necessidade de uso de um computador pessoal durante as aulas teóricas e práticas e a motivação para um maior uso de TDICs.

O projeto de pesquisa foi submetido ao Comitê de Ética em Pesquisa (CEP) e aprovado pelo Parecer no 2.403.385, de 28 de novembro de 2017, CAAE 78899617.2.0000.5108. Foram utilizados questionários autoaplicáveis juntamente com o Termo de Consentimento Livre e Esclarecido (TCLE).

A pesquisa de campo foi dividida em 2 etapas. $\mathrm{Na} 1^{\mathrm{a}}$ etapa foram aplicados questionários via plataforma online. A população total de docentes, composta por 48 pessoas, foi convidada a participar do estudo. Durante a execução da pesquisa de campo não havia docentes afastados ou em regime substituto, assim toda a equipe de professores da FAMED, com exceção da autora deste trabalho, foi convidada a participar do estudo. Aqueles que concordaram em participar assinaram o TCLE e receberam por e-mail um link de acesso ao questionário, disponibilizado em plataforma online. Esta etapa aconteceu nos meses de dezembro de 2017 e janeiro de 2018.

A $2^{\mathrm{a}}$ etapa consistiu na aplicação de questionários em papel para os docentes que informassem que ainda não tiveram respondido via plataforma. Esta etapa aconteceu nos meses de fevereiro e março de 2018.

Os riscos a que o participante estava exposto foram mínimos e envolvem a dimensão moral. Existia possibilidade de constrangimento ao responder o questionário, desconforto e quebra de sigilo. Estes riscos foram minimizados pelos seguintes procedimentos: esclarecimento prévio sobre a pesquisa, leitura do TCLE, participação voluntária e possibilidade de interromper o preenchimento do questionário a qualquer momento. Além disso, as respostas foram confidenciais, o questionário não foi identificado pelo nome, para que fosse mantido o anonimato. Os benefícios relacionados a participação envolviam o direcionamento de medidas para melhoria do ensino, contato com TDICs possivelmente desconhecidas e possibilidade do desenvolvimento de futuros planos de incentivo ao uso das TDICs pela FAMED.

Após o término da pesquisa de campo foi feita a análise de conteúdo dos questionários. A abordagem dos dados foi feita de forma quali-quantitativa. A pesquisa qualitativa se diferencia da quantitativa pela forma de abordagem de uma realidade e da maneira pela qual os dados são tratados e analisados. O método qualitativo não aplica instrumentos estatísticos para análise de um problema, uma vez que seu objetivo não é medir nem numerar os eventos estudados (RICHARDSON, 2008). De acordo com Creswell (2010), o desenvolvimento e a legitimidade percebida de ambos os métodos geraram uma popularização de pesquisas quali-quantitativa, que abrangem os pontos fortes tanto da abordagem 
qualitativa quanto da quantitativa, proporcionando uma maior compreensão dos problemas estudados.

Para determinar o perfil demográfico e profissional dos docentes, perceber como eles inserem as tecnologias em suas práticas pedagógicas e como o projeto pedagógico do curso favorece o uso das tecnologias na docência, foi feita análise quantitativa das respostas por meio de gráficos de barras e setores. Para verificar a existência de associação entre os fatores investigados e a quantidade de ferramentas de TDICs usadas pelos docentes em ambiente profissional, foi realizada análise quantitativa, através de análise estatística exploratória e de testes estatísticos.

Na análise exploratória foram utilizados gráficos de caixas e de dispersão para visualização dos dados e suas relações. Posteriormente foram feitos testes estatísticos não paramétricos, devido ao baixo número de observações, especialmente quando se formavam agrupamentos. Segundo Viera (2010) uma vantagem dos testes não paramétricos é menor sensibilidade à presença de outliers. O teste de Shapiro-Wilk foi ainda utilizado para interpretar a distribuição do numero de TDICs, para fins profissionais e para a docência.

O programa e linguagem estatística "R" (R DEVELOPMENT CORE TEAM, 2018) foi utilizado para a análise dos dados. Os testes estatísticos foram realizados considerando um nível de significância de 0,05 para verificar associações entre as variáveis.

Existem testes que podem ser utilizados para ajudar a definir se existe uma distribuição normal. Um exemplo destes testes é o Shapiro-Wilk. Este teste pode ser calculado facilmente em programas de análise estatística, como o $\mathrm{R}$, a partir de comandos já definidos. Neste caso, consideramos uma hipótese nula de que os valores seguem uma distribuição normal. Para um grau de confiança de 95\%, aceitamos a hipótese alternativa de que os dados não seguem uma distribuição normal se o p-valor é menor que 0,05 (UGARTE; MILITINO; ARNHOLT, 2015).

O Teste de Kruskal-Wallis foi usado para testar a hipótese nula de que os grupos de docentes categorizados possuem funções de distribuição iguais em relação ao número de TDICs para uso profissional contra a hipótese alternativa de que ao menos dois dos grupos possuem distribuição diferente em relação a esse item.

A análise de regressão quantílica foi utilizada inicialmente para estimar o número de TDICs utilizadas pela mediana dos docentes nas atividades profissionais, a partir dos números de TDICs utilizadas pessoalmente. Posteriormente, também foi utilizado esse teste para criar um modelo que estima o número de TDICs utilizadas pela mediana dos docentes nas atividades profissionais a partir de todos fatores que sugeriram relação significativa durante a abordagem quantitativa dos dados.

O Teste de Nagelkerke foi utilizado para obter o percentual de variações explicadas por um modelo que estima o número de TDICs utilizadas pelos docentes a partir das interferências dos fatores considerados determinantes. 


\section{Resultados e Discussão}

Conforme informações fornecidas pela coordenação do curso, a expectativa para os próximos anos é que o curso tenha ao todo 55 docentes. O corpo docente da FAMED ainda está se formando e assumindo sua identidade. No momento em que foi feita a pesquisa de campo era composto por 48 docentes. Dos 48 docentes convidados para participar do estudo, 42 responderam ao questionário.

A maioria dos docentes possui faixa etária entre 36 e 40 anos (Figura 1).

Figura 1 - Gráfico do perfil sociodemográfico dos docentes da FAMED, por idade, em março de 2018

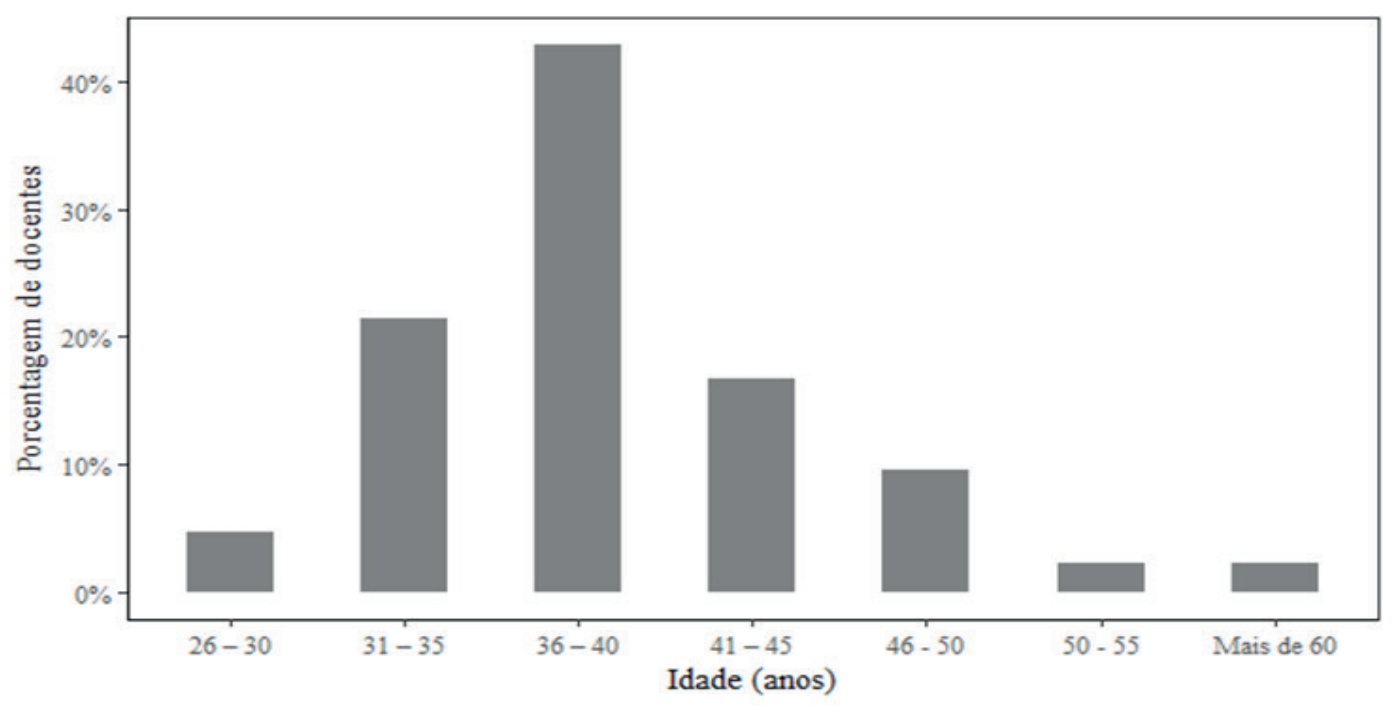

Fonte: Elaborado pela autora.

A maioria é do sexo feminino (64\%). Mesmo avaliando separadamente os docentes médicos e não-médicos da FAMED, observa-se uma prevalência de profissionais do sexo feminino em ambos os grupos. O fato de a maior parte dos professores da FAMED serem do sexo feminino pode estar associado ao fato de ser uma instituição nova, com profissionais de idade mais jovem.

Ferreira e Souza (2016), realizaram um estudo que obteve como resultado em uma maioria de professores-médicos do sexo masculino. Eles reforçaram o achado com dados da Comissão Interinstitucional Nacional de Avaliação do Ensino Médico (CINAEM), evidenciando que a maioria dos professores médicos no Brasil são do sexo masculino. $O$ fato da maioria dos docentes ter a titulação de especialista está relacionado com o fato da maioria ser médico.

Segundo o Conselho Regional de Medicina de Minas Gerais (CRMMG), atualmente os homens são maioria entre os médicos no Brasil, porém a diferença em relação ao número de mulheres vem reduzindo a cada ano e o sexo feminino já predomina na faixa etária 
de 30 a 34 anos. A média de idade dos médicos no país também vem caindo e atualmente é de 45,4 anos (CRMMG, 2018).

Na Figura 2 está apresentada a quantidade de docentes por titulação.

Figura 2 - Gráfico da quantidade de docentes por titulação, em março de 2018

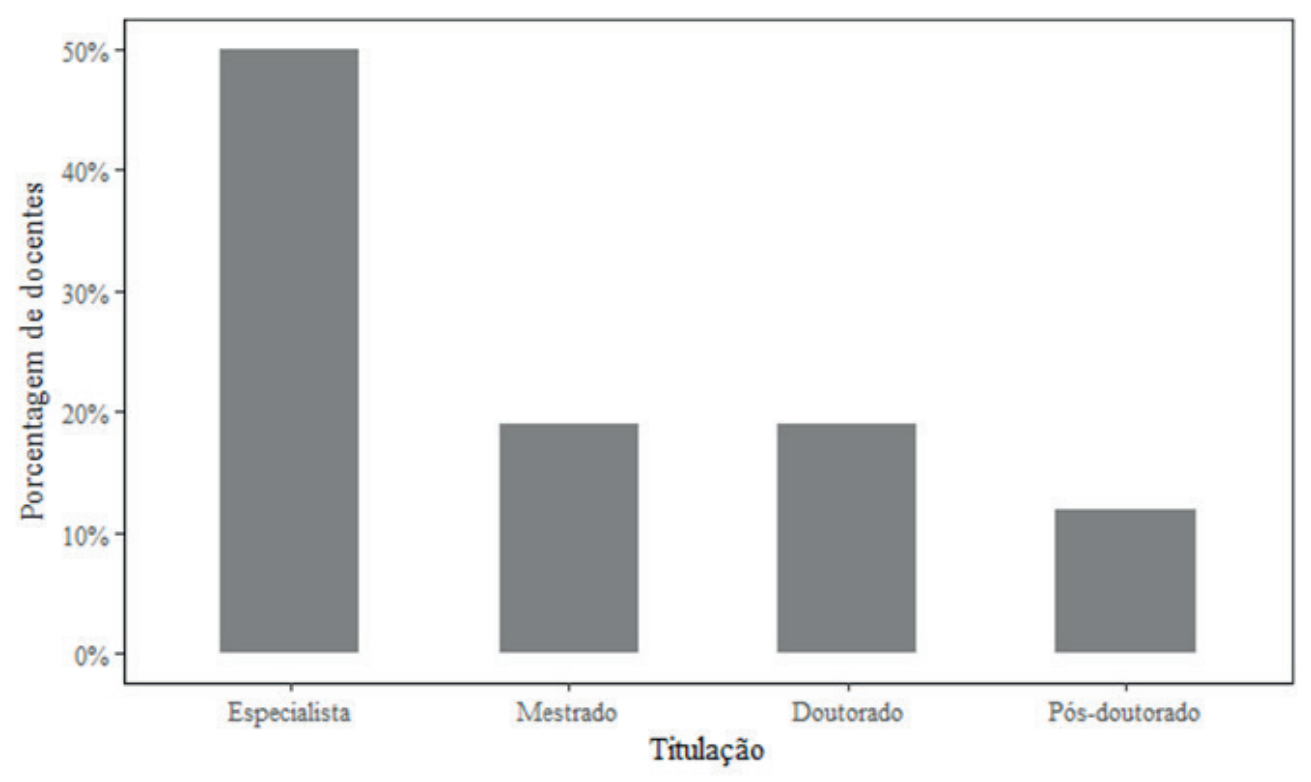

Fonte: Elaborado pela autora.

O fato da maioria dos docentes ter a titulação de especialista está relacionado com o fato da maioria ser médico (Figura 3).

Figura 3 - Gráfico da quantidade de docentes por formação, em março de 2018

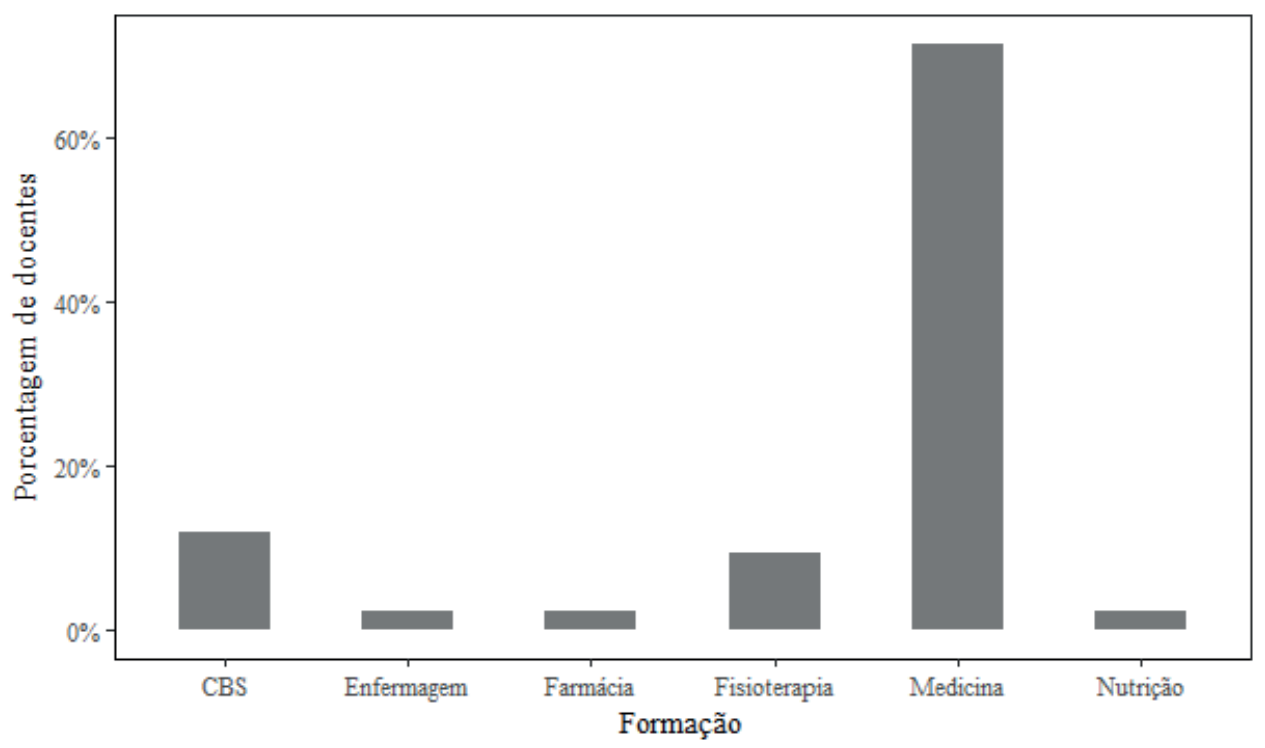

Fonte: Elaborado pela autora. 
Em geral as capacitações médicas são voltadas para especializações e não para capacitação acadêmica. Em estudo realizado por Ferreira e Souza (2016), a maior parte dos médicos-professores também afirmou ter titulação especialista, mas apenas metade possuía mestrado. Neste estudo os docentes consideraram as matérias do mestrado ou da especialização que cursaram como uma forma de qualificação em educação.

Segundo CRMMG (2018) apenas 38\% dos médicos não possuem título de especialista e este número vem diminuindo devido a expansão de vagas nos programas de residência médica.

O professor médico em geral, não está vinculado ao regime de dedicação exclusiva e exerce a docência no tempo que resta de suas atividades clínicas. Segundo Ferreira e Souza (2016) o tempo é como fator limitante para o exercício da docência pelo médico, em virtude das diversas atividades desenvolvidas em paralelo fora das instituições de ensino.

Ferreira e Souza (2016) também observaram que a maior parte dos professores não se dedicavam a profissão docente em tempo integral. Segundo os autores, estes dados estão de acordo com os dados da CINAEM e essa realidade se justifica pelos baixos salários pagos aos professores. A diferença salarial entre a remuneração como professor e como profissional médico pode tornar a docência um exercício menos atrativo aos médicos. Apesar disso a presença destes nos cursos de graduação em medicina é fundamental.

A quantidade de TDICs utilizadas pelos docentes, para uso pessoal, estão representadas na Figura 4.

Figura 4 - Gráfico da quantidade de docentes por TDICs utilizada para uso pessoal, em março de 2018

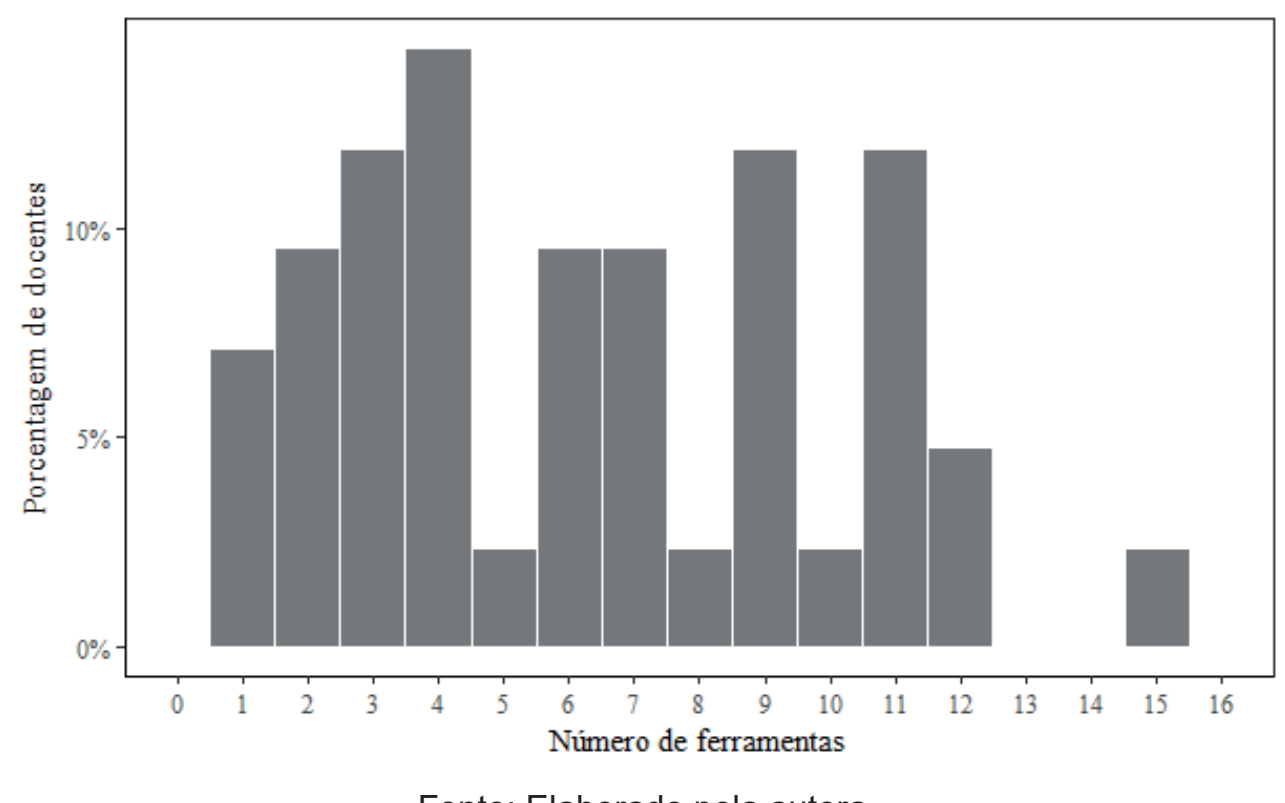

Fonte: Elaborado pela autora.

Percebe-se que a maior parte dos docentes possui conhecimentos que tornam possível o uso de uma ou mais TDICs. Na Figura 4 não se observa uma distribuição normal e simétrica, do número de TDICs utilizadas para fins pessoais, em volta da média. O teste de normalidade 
de Shapiro -Wilk para esta variável aceitou a hipótese alternativa de que não há uma distribuição normal ( $p$-value $=0,03798$ ). As formas de uso pessoal das TDICs estão representadas na Tabela 1. As ferramentas mais utilizadas para fins pessoais foram o envio e recebimento de arquivos por e-mail (39), a pesquisa em bases de dados (32) e o acesso a sites de interesse pessoal (31).

Tabela 1- Principais TDICs utilizadas para uso pessoal, em março de 2018

\begin{tabular}{lll}
\hline TDICs para uso pessoal & Número de docentes & Percentual (\%) \\
\hline Enviar e receber arquivos por e-mail & 39 & 92,86 \\
\hline Pesquisa em base de dados & 32 & 76,19 \\
\hline Acessar sites específicos & 31 & 73,81 \\
\hline Criar apresentações & 21 & 50,00 \\
\hline Rede Social & 18 & 42,86 \\
\hline Enviar vídeos & 12 & 28,57 \\
\hline Responder questionários online & 14 & 33,33 \\
\hline Upload materiais & 24 & 57,14 \\
\hline Computadores da UFVJM & 9 & 21,43 \\
\hline Fórum & 5 & 11,90 \\
\hline Videoconferência & 5 & 11,90 \\
\hline Realizar cursos a distância & 15 & 35,71 \\
\hline Construir de textos em ambiente virtual & 6 & 14,28 \\
\hline Criar, editar ou gerenciar Blogs & 1 & 2,38 \\
\hline Download de materiais & 17 & 40,48 \\
\hline Programas de simulação realística & 4 & 9,52 \\
Chat & 2 & 4,76 \\
\hline
\end{tabular}

Fonte: Elaborado pela autora.

A quantidade de TDICs utilizadas pelos docentes, para uso profissional, está representada na Figura 5.

Figura 5 - Gráfico da quantidade de docentes por TDICs utilizadas para uso profissional, em março de 2018

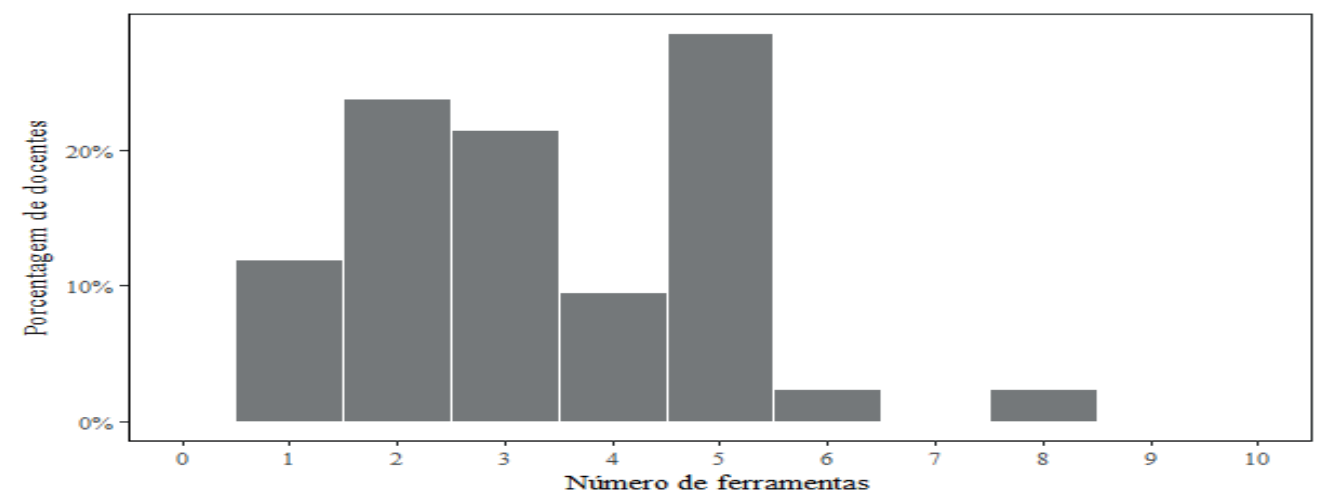

Fonte: Elaborado pela autora. 
Percebe-se que todos os docentes utilizam pelo menos uma TDIC na docência. A média de ferramentas utilizadas na docência foi 2,9, ou seja, inferior à média do número de ferramentas para uso pessoal (6). Na figura 5 também não se observa uma distribuição normal do número de TDICs utilizadas para fins profissionais. $O$ teste de normalidade de Shapiro -Wilk para esta variável aceitou a hipótese alternativa de que não há uma distribuição normal ( $p$-value $=0.003252$ ).

As ferramentas mais utilizadas foram enviar ou receber de arquivos por e-mail (31), disponibilização de materiais para download do aluno em ambiente virtual (19) e sugestão de sites específicos em saúde para leitura pelos alunos (18). Ao comparar com as estratégias utilizadas para fins pessoais, percebe-se que há uma tendência em utilizar em sala de aula as mesmas estratégias de uso pessoal.

A maior parte dos docentes não realizou curso de capacitação em informática e dos que realizaram, foi por motivo pessoal. Ao contrário de capacitação em informática, a maioria dos docentes realizou algum curso de capacitação de docência após ingresso na FAMED.

As Metodologias Ativas de Ensino e Aprendizagem (MAEA), são parte dos métodos de ensino inovadores propostos no projeto pedagógico do curso. Este método parece favorecer o uso das TIDCs, na opinião dos docentes (Tabela 2).

Tabela 2 - Formas de favorecimento para o uso das tecnologias pela FAMED, na percepção dos docentes (pergunta aberta), em março de 2018

\begin{tabular}{lll}
\hline $\begin{array}{l}\text { Estratégias para favorecimento do uso } \\
\text { das tecnologias pela FAMED }\end{array}$ & Número de docentes & $\begin{array}{l}\text { Percentual do total de } \\
\text { docentes (\%) }\end{array}$ \\
\hline MAEA & 9 & 21,4 \\
\hline Aprimoram o conhecimento & 2 & 4,8 \\
\hline Possibilidade de uso do EaD & 4 & 9,5 \\
\hline $\begin{array}{l}\text { Disponibilizando ferramentas (moodle e laboratório de } \\
\text { informática) }\end{array}$ & 6 & 14,3 \\
\hline A partir de turmas reduzidas & 1 & 2,4 \\
Facilitando a comunicação aluno docente & 2 & 4,8 \\
\hline
\end{tabular}

Legenda: MAEA: Metodologias Ativas de Ensino e Aprendizagem

Fonte: Elaborado pela autora.

Quando se compara as respostas desta questão como as estratégias mais utilizadas em sala (Tabela 2), percebe-se que a pesquisa em base de dados foi uma das mais citadas. Isto está de acordo com a proposta da FAMED, de oferecer um ensino voltado para o aluno, tendo o professor como mediador do conhecimento que está sendo descoberto pela turma e as TDICs como um facilitador do aprendizado.

A indisponibilidade dos equipamentos em sala é o principal obstáculo para o uso das tecnologias em sala (Tabela 3). 
Tabela 3 - Dificuldades para maior uso das TDICs durante as aulas na FAMED (março de 2018)

\begin{tabular}{lll}
\hline Dificuldades para maior uso das TDICs nas aulas & Numero de docentes & Percentual (\%) \\
\hline Indisponibilidade de equipamentos & 21 & 50,00 \\
\hline Pouca habilidade com os equipamentos & 17 & 40,48 \\
\hline Falta de tempo & 5 & 11,90 \\
\hline Pouco treinamento didático & 18 & 42,86 \\
Não tenho dificuldades & 3 & 7,14 \\
\hline
\end{tabular}

Fonte: Elaborado pela autora.

Como ainda não existe uma estrutura de sala de aulas definitiva, as aulas teóricas são geralmente ministradas no pavilhão de aulas, cabendo ao docente levar para cada aula materiais como projetor multimídia, cabo de internet, aparelho de som, seu laptop pessoal e demais itens que considerar necessário. A construção de uma estrutura definitiva para o curso de medicina pode facilitar o controle desses materiais, favorecendo diferentes estratégias pedagógicas.

Os resultados indicaram que a maior parte dos docentes não possui acesso a computador nas aulas ministradas na rede do SUS. Os docentes que utilizam computador pessoal em sala o fazem mais pela indisponibilidade do mesmo do que por preferência.

Segundo Santos (2016), tempo para planejar as aulas, indisponibilidade do equipamento na instituição, falta de habilidade para manusear as ferramentas e pouco treinamento didático estão entre os possíveis motivos que podem servir de obstáculos para o uso das TDICs como recurso didático.

Os gráficos de caixa evidenciaram a existência de associação entre alguns dos fatores e a quantidade de ferramentas de TDICs usadas pelos docentes no ambiente profissional. Embora visualmente seja possível perceber o maior uso de ferramentas com o maior tempo de atuação (Figura 6), não foram encontradas evidências estatísticas ( $p$-valor $=0,1179$ ).

Figura 6 - Gráfico de Caixas: Quantidade de TDICs utilizadas para uso profissional e tempo de atuação na FAMED

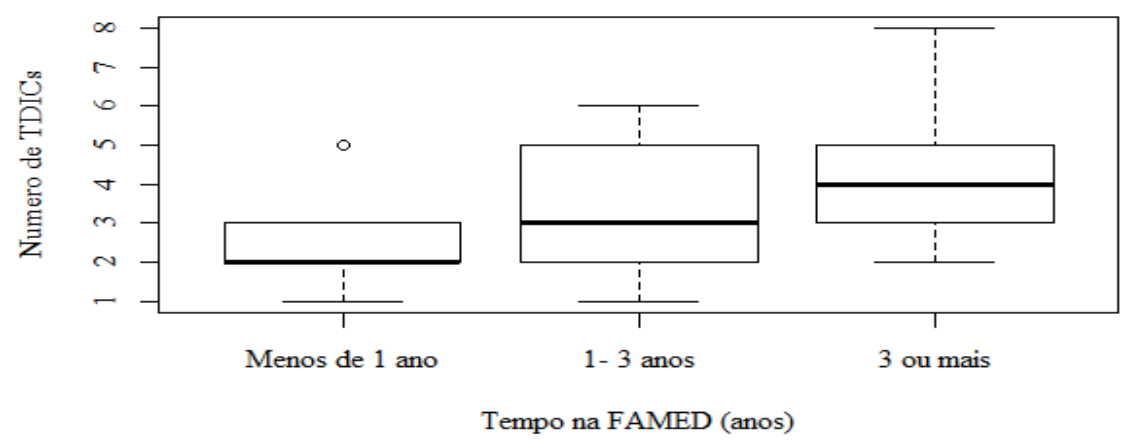

Fonte: Elaborado pela autora. 
O mesmo ocorre ao se comparar o uso de ferramentas com a maior titulação ( $p$-valor $=0,232)$ e idade ( $p$-valor $=0.1095)$. Este resultado contradiz o encontrado por Pereira et al. (2016), quando mostrou haver associação do número de instrumentos utilizados com a titulação e idade.

Na Figura 7 está apresentado o gráfico da Quantidade de TDICs utilizadas para uso profissional e impressão de favorecimento.

Figura 7 - Gráfico de Caixas: Quantidade de TDICs utilizadas para uso profissional e impressão de favorecimento

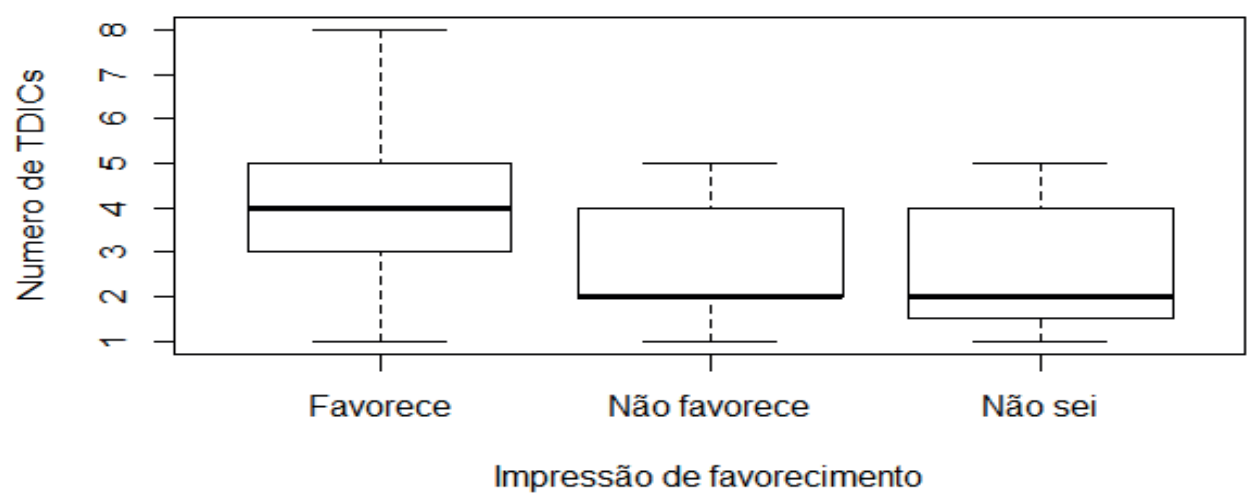

Fonte: Elaborado pela autora.

A impressão dos docentes sobre se sentirem estimulados diante das estratégias adotadas pelo curso, se mostrou estatisticamente significativa para um maior uso das tecnologias ( $p$-valor $=0,03225$ ). Já disponibilidade de equipamentos na rede do SUS não apresentou relação estatisticamente significativa com o maior uso de ferramentas pelos docentes ( $p$-valor $=0,3977$ ), assim como a disponibilidade de TDICs em sala ( $p$-valor = 0,8015).

Este resultado é diferente do encontrado por Panda e Mishra (2007). Os autores relatam que as principais barreiras para educação mediada pela tecnologia incluem ausência de acesso à internet e falta de treinamento.

Conforme pode ser observado na Figura 8, A realização de algum curso de iniciação à docência também não apresentou relação estatisticamente significativa com o maior uso de ferramentas pelos docentes ( $p$-valor $=0,5056)$. Este resultado também contradiz o encontrado por Pereira et al. (2016), quando mostrou haver associação do número de instrumentos utilizados com a participação em cursos voltados a preparação para docência. 
Figura 8 - Gráfico de Caixas: Quantidade de TDICs utilizadas para uso profissional e realização algum curso de iniciação à docência

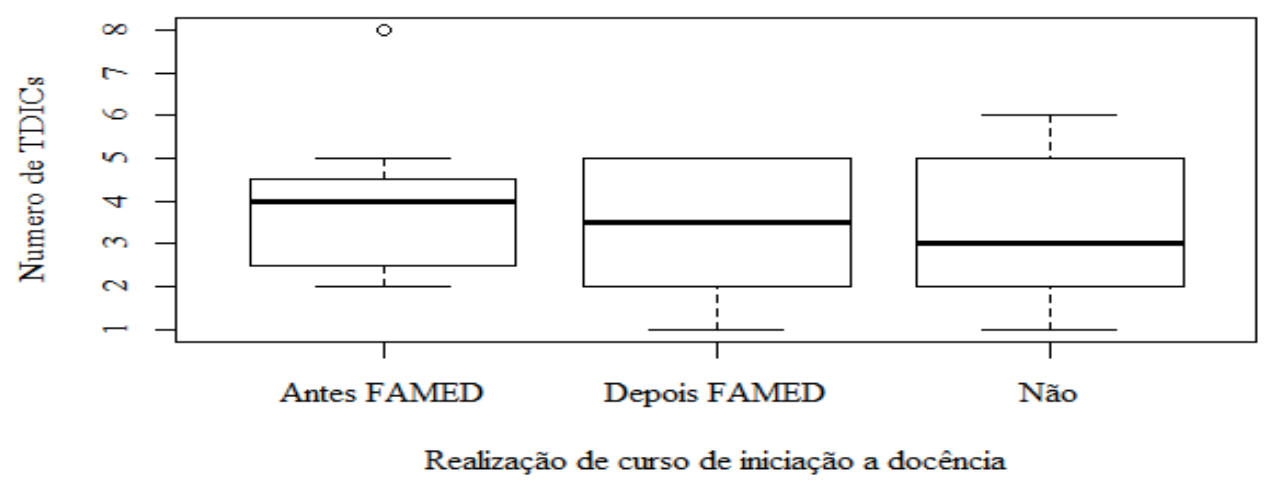

Fonte: Elaborado pela autora.

Em contrapartida este resultado também reafirma o que foi percebido por Ferreira e Souza (2016). Os autores observaram que os saberes docentes são construídos com as vivências de cada professor, e não necessariamente por meio de cursos específicos. Para os pesquisados, ter ou não uma formação docente específica não é um pré-requisito para ser um "bom" professor de medicina.

Na Figura 9 está apresentado o gráfico da quantidade de TDICs utilizadas para uso profissional e motivação.

Figura 9 - Gráfico de Caixas: Quantidade de TDICs utilizadas para uso profissional e motivação

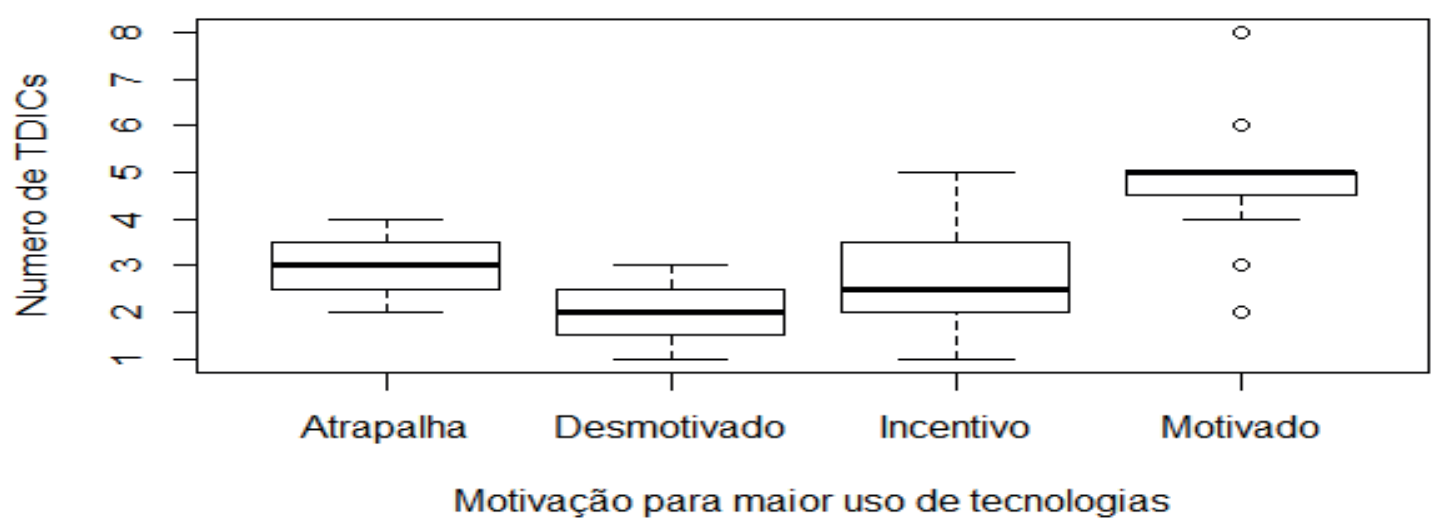

Fonte: Elaborado pela autora. 
A análise da variável a motivação pessoal, se mostrou estatisticamente significativa para o maior uso de ferramentas. Sugere-se que independentemente de quais políticas são adotadas pela FAMED no sentido de incentivar os docentes em suas práticas pedagógicas, a motivação pessoal é um dos principais fatores determinantes para o maior uso de TDICs na docência ( $p$-valor $=0,0007773$ ).

Ao se observar o grupo dos docentes que sentem motivados a utilizar mais tecnologias, percebe-se a presença de outliers. Ou seja, alguns dos professores divergem muito do restante do grupo analisado, para mais ou menos TDICs na docência, não seguindo o padrão habitual. Uma vantagem dos testes não paramétricos, como o utilizado nesta análise, é a menor sensibilidade a estes ouliers. Este resultado é semelhante ao encontrado por Panda e Mishra (2007). Os autores perceberam que um dos principais motivadores para educação mediada pela tecnologia é o interesse pessoal.

Na Figura 10 está apresentada a relação entre a quantidade de TDICs utilizadas para uso profissional e quantidade de TDICs utilizadas para uso pessoal, através de um gráfico de dispersão.

Figura 10 - Gráfico de Dispersão: Quantidade de TDICs utilizadas para uso profissional e Quantidade de TDICs utilizadas para uso pessoal

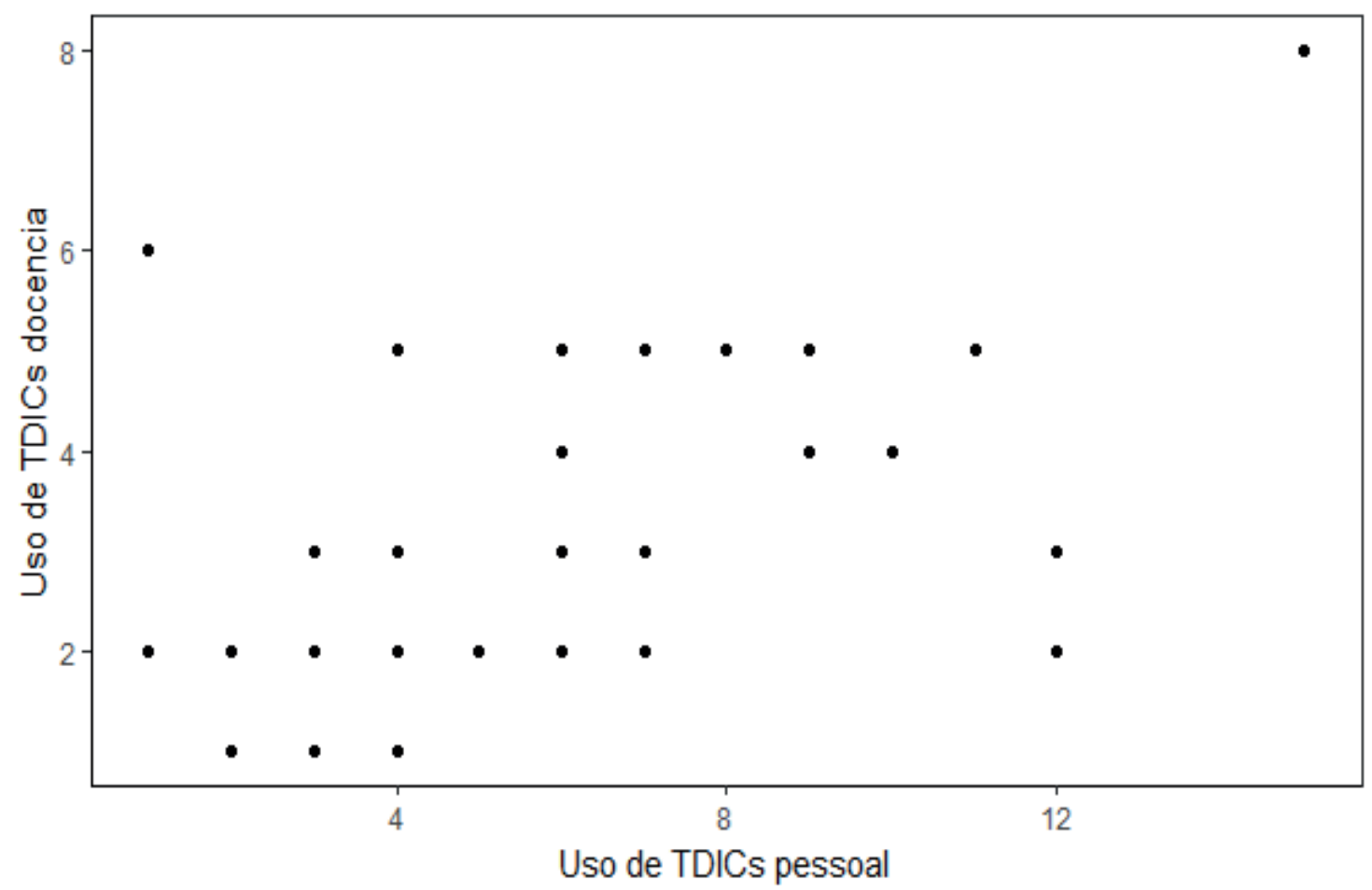

Fonte: Elaborado pela autora.

Ao se observar a Figura 10, percebe-se que há uma tendência positiva entre o uso pessoal e o uso profissional. A análise de regressão quantílica que estima o número de 
TDICs utilizado pela mediana dos docentes a partir do número de TDICs pessoal é apresentado na Tabela 4.

Tabela 4 - Modelagem do uso de TDICs na vida pessoal para estimar o uso de TDICs na docência

\begin{tabular}{llll}
\hline Fator & Coeficiente & Limite Inferior & Limite Superior \\
\hline Intercepto & 1,33 & 0,42 & 2,25 \\
\hline Número TDICs pessoal & 0,33 & 0,20 & 0,36 \\
\hline
\end{tabular}

Fonte: Elaborado pela autora.

Este modelo sugere que um docente da FAMED tende a utilizar 1,3 TDICs na docência. Para cada TDIC utilizada para fins pessoais, aumenta-se 0,33 TDICs na docência. Este modelo foi capaz de explicar $53 \%$ da variância presente nos dados, pelo Teste de Nagelkerke ( $p$-valor $=2.4364 \mathrm{e}-08)$.

A análise de regressão quantílica dos dados que se mostraram significativos, permitiu criar um modelo estimativo do número de TDICs esperado que um docente utilize em suas práticas pedagógicas (Tabela 5 ).

Tabela 5 - Modelagem dos fatores determinantes para o uso de TDICs na docência. Variáveis em negrito não contém o zero no intervalo dos limites

\begin{tabular}{llll}
\hline Fator & Coeficiente & Limite Inferior & Limite Superior \\
\hline Intercepto & 2,2 & -0.65 & 2.94 \\
\hline Número TDICs pessoal & 0,2 & 0,09 & 0,35 \\
\hline Sentir desmotivado & $-0,6$ & -1.07 & 2.13 \\
\hline Sentir incentivado & 0 & -0.25 & 2.28 \\
\hline Sentir motivado & 1,2 & 1,16 & 2,93 \\
\hline Impressão de não favorecimento & $-0,6$ & $-1,84$ & $-0,44$ \\
\hline Não saber se é favorecido & $-1,6$ & $-2,50$ & $-0,61$ \\
\hline
\end{tabular}

Fonte: Elaborado pela autora.

A análise regressão quantílica mostra que um docente da FAMED tende a utilizar 2,2 TDICs na docência. Para cada TDIC utilizada para fins pessoais, aumenta-se 0,2 TDICs na docência. Caso se sinta motivado, o impacto é de 1,2 TDICs a mais na docência. Caso não se sinta favorecido pelas estratégias da FAMED, há uma redução de 0,6 no número de TDICs utilizadas na docência. Docentes indecisos quanto ao favorecimento ou não tem um impacto negativo ainda maior, reduzindo em 1,6 TDICs usadas na docência. Este modelo foi capaz de explicar $74 \%$ da variância presente nos dados, pelo Teste de Nagelkerke (p-valor $=5,3308 \mathrm{e}-10)$. Ou seja, este modelo é mais representativo do que o modelo anterior, que 
considera apenas as TDICs pessoais. As variáveis destacadas em negrito mostram maior significância por não conterem zero no intervalo de confiança dos coeficientes.

Sugere-se que independentemente de quais políticas sejam adotadas pela FAMED no sentido de incentivar os docentes em utilizar mais tecnologias em suas práticas pedagógicas, a motivação pessoal, a impressão de sentir apoio institucional e as características pessoais do professor são os principais fatores determinantes para o maior uso de TDICs na docência.

\section{Conclusão}

O perfil demográfico e profissional atual do corpo docente da FAMED, se mostrou composto em sua maioria, por médicos, não vinculados ao regime de dedicação exclusiva. Isso torna o fator tempo de preparo das aulas, algo a ser considerado.

As tecnologias podem ser aparentemente mais trabalhosas no início, porém, a longo prazo, elas são facilmente adaptadas as novas turmas. Isso as torna vantajosas a um perfil de docentes com pouco tempo. A medida que a equipe de professores for se completando e que as primeiras turmas forem se formando, os docentes tendem a se fixar em determinados conteúdos. Diante disso, há uma perspectiva favorável para o uso cada vez maior de TDICs no ensino e aprendizagem nos próximos anos.

Ao se comparar a FAMED com outras instituições que oferecem a graduação em medicina, é notável a diferença etária e o tempo de atuação dos docentes. O fato de ser um curso já criado nos moldes atuais de ensino e aprendizagem coloca esta instituição em vantagem sobre instituições mais antigas, que possuem o desafio de modificar um currículo tradicional já consolidado.

A existência de uma aptidão prévia dos futuros professores com as tecnologias, antes do ingresso na docência, se mostrou um fator decisivo para uma maior adequação das atividades docentes desenvolvidas as necessidades do curso. Assim, esta característica deve ser levada em consideração nas futuras seleções de docentes.

Os resultados deste estudo apontam para a importância se observar como os candidatos dos concursos e processos seletivos em andamento valorizam as TDICs durante as avaliações. Em geral as seleções para professor de magistério superior contavam com formas de avaliação que incluem prova didática, prova prática; avaliação de títulos e apresentação oral do projeto de atividades acadêmicas. Em todas estas modalidades é possível avaliar a afinidade já existente dos candidatos com o uso das TDICs no ensino e incluir este tópico como um dos quesitos a serem avaliados.

Nas provas didática e escrita, sugere-se incluir "o uso adequado dos recursos didáticos" como um tema transversal transpassando todos os conteúdos específicos do programa. Na prova prática, são sugeridas situações que representam possíveis problemas práticos do dia-a-dia da docência para que o candidato ofereça uma solução, no sentido de direcionar o candidato para desenvolver soluções que envolvam o ensino a distância. Por 
exemplo: "Durante o atendimento em um posto de saúde em Diamantina, os alunos percebem que o paciente deseja parar de fumar, porém há dúvidas sobre qual seria a melhor conduta. Existem apenas telefones celulares pessoais com acesso a internet disponíveis para consulta. Como os alunos poderiam ser direcionados na busca pela melhor conduta?". $\mathrm{Na}$ avaliação de títulos, sugere-se avaliar a realização de cursos de capacitação em educação mediada por tecnologias e a apresentação de trabalhos em eventos científicos sobre a tecnologia no ensino. Na apresentação oral do projeto de atividades acadêmicas sugere-se adequar o item o que se refere a "relevância e atualidade da proposta de trabalho" para "relevância e uso de tecnologias na proposta de trabalho".

No sentido de contribuir com a motivação dos docentes já em exercício, sugere-se que as comissões que avaliam os critérios de pontuação para estágio probatório e progressão docente considerem maior favorecimento da formação dos professores em TDICs. A produção ou utilização de tecnologias no ensino poderia ser avaliada no item "capacidade de inovar e tomar decisões no exercício do cargo", no estágio probatório, e nos tópicos "produção intelectual tecnológica" e "desempenho e capacitação docente", na tabela de progressão. O Instrumento de Avaliação do Ensino, preenchido pelos discentes a cada semestre letivo, poderia incluir uma pergunta envolvendo o uso adequado do ambiente moodle e outras tecnologias pelo docente avaliado.

Apesar das mudanças sugeridas, a "Pedagogia Tradicional" não é necessariamente ruim. Ela foi eficaz por muitos anos e é defendida por muitos autores nos dias de hoje. $O$ professor deve ter autonomia para definir qual a metodologia mais adequada ao seu perfil, aos seus alunos e aos objetivos de aprendizagem. Ele deve ter autonomia para utilizar o blended learning, ou seja, um misto de várias metodologias. Isso envolve misturar o ensino tradicional com as metodologias ativas assim como ensino presencial e com o ensino a distância e assim como o uso e não uso das TDICs.

Espera-se que os resultados e reflexões apresentados possam levar os docentes e gestores a refletirem sobre suas práticas de forma a estimular o maior uso de TDICs. 


\section{Referências}

ALONSO, C. A. Internet no Brasil - alguns dos desafios a enfrentar. Informática Pública, v. 4, n. 2 , p. 169-184, 2002.

BECKER, F. O que é construtivismo. Revista de educação AEC. v. 21, n. 83, p7-15. Brasília, 1992.

BRASIL. Resolução No 3, DE 20 DE JUNHO DE 2014. Diretrizes Curriculares Nacionais dos Cursos de Graduação em Medicina. Brasília: Ministério da Educação: Conselho Nacional da Educação, 2014.

CARLINI, A. L. O professor do ensino superior e a inclusão digital. In: CARLINI, A. L. SCARPATO, M. Ensino superior: questões sobre a formação do professor. São Paulo: Avercamp; p. 83-94. 2008.

CRESWELL, J. W. Projeto de pesquisa métodos qualitativo, quantitativo e misto. In: Projeto de pesquisa métodos qualitativo, quantitativo e misto. 2010.

CRMMG - CONSELHO REGIONAL DE MEDICINA DE MINAS GERAIS. Demografia Médica. Jornal do CRM-MG. v.64, p.13-14, 2018.

DEBALD, F. R. B. TICs e a prática pedagógica universitária. Pleiade, v.1, n.1, p83-94, 2007.

DOWBOR, L. Tecnologias do conhecimento: os desafios da educação. São Paulo: Vozes, 2013.

ELIADEMY. Criação de curso online. Disponível em: https://eliademy.com/pt_br/. Acesso em 04 jan. 2018.

FERREIRA, C. C.; SOUZA, A. M. L. Formação e Prática do Professor de Medicina: um Estudo Realizado na Universidade Federal de Rondônia. Revista Brasileira de Educação Médica. v. 40, n.4, p.635-643, 2016

KHANACADEMY. Ambiente de aprendizado digital para alunos e professores. Disponível em: <https://pt.khanacademy.org>. Acesso em: 04 jan. 2018.

LAU, F. A.; MENDES, V. F.; VENTURA; A. A.; BOLLELA, V. R.; TEIXEIRA, L. A. S. Implantação de Estratégias de Ensino à Distância durante o Internato: Desafios e Perspectivas. Revista Brasileira de Educação Médica. v.41,n.2, p. 269-277; 2017

LOPES, R. P. Formação para uso das Tecnologias Digitais de Informação e Comunicação nas licenciaturas das universidades estaduais paulistas. Dissertação (Mestrado em Educação) - Universidade Estadual Paulista, Faculdade de Ciências e Tecnologia. Presidente Prudente, SP, 2010. 224 p.

MEDRONHO, R.A.; BLOCH, K.V.; LUIZ, R.R.; WERNECK G.L. Epidemiologia. 2ed. p.176-177. São Paulo: Atheneu, 2009.

MIRANDA, G. L. Limites e possibilidades das TIC na educação. Sísifo - Revista de Ciências da Educação, n. 3, p. 41-50, 2007. Disponível em: <http://sisifo.fpce.ul.pt>. Acesso em: 12 dez. 2016. 
MORAN, J. Mudando a educação com metodologias ativas. Coleção Mídias Contemporâneas. Convergências Midiáticas, Educação e Cidadania: aproximações jovens. Vol. 2. p15- 31. PROEX/ UEPG, 2015.

OLIVEIRA, N. A.; MEIRELLES, R. M. S.; CURY, G. C.; ALVES, L. A. Mudanças Curriculares no Ensino Médico Brasileiro: um Debate Crucial no Contexto do Promed. Revista Brasileira de Educação Médica. 32 (3): 333-346; 2008.

PANDA, S. MISHRA, S. E-learning in a Mega Open University: faculty atitude, barriers and motivators. Educational Media International, v.44, n.4, 2007.

PEREIRA, T. A.; ARECO, K. C. N.; TARCIA, R. M. L.; SIGULEM, D. Uso das Tecnologias de Informação e Comunicação por Professores da Área da Saúde da Universidade Federal de São Paulo. Revista Brasileira de Educação Médica. vol. 40, p. 59-66. Rio de Janeiro, 2016.

R DEVELOPMENT CORE TEAM.R: A language and environment for statistical computing. R Foundation for Statistical Computing, Vienna, Austria, 2018. ISBN 3-900051-07-0, URL http://www.R-project.org.

RICHARDSON, R. J. Pesquisa Social: Métodos e Técnicas. $3^{a}$ edição. São Paulo, Atlas, 2008. 334 p.

SEPTRIS. Página do jogo. Disponível em: <http://med.stanford.edu/septris/game/Septris.html>. Acesso em: 05 dez. 2016.

STANFORD UNIVERSITY. Medical school. Information resources and technology. Palo Alto, CA. Estados Unidos.

Disponível em: http://med.stanford.edu/irt.html. Acesso em 05 dez. 2016.

UFVJM - UNIVERSIDADE FEDERAL DOS VALES DO JEQUITINHONHA E MUCURI. Projeto pedagógico do curso de graduação em medicina - Diamantina. Diamantina: UFVJM, Faculdade de Medicina, 2017. 135 p.

UNITED NATIONAL EDUCATIONAL. Scientific and Cultural Organization. Information and Communication Technologies in Schools: A Handbook for Teachers or How ICT Can Create New, Open Learning Environments. UNESCO, Division of Higher Education. Paris, 2015.

UGARTE, M. D.; MILITINO, A. F.; ARNHOLT, A.T. Probability and Statistics with R. 2ed. 648p. CRC Press/Chapman and Hall, 2015.

VIEIRA, S. Bioestatística: Tópicos Avançados. 3a . ed. p.161-162. Rio de Janeiro: Elsevier Brasil, 2010.

WHATSAPP. Aplicativo para telefones. Disponível em: <https://www.whatsapp.com/?l=pt_br>. Acesso em: 28 de jun. 2018. 